\title{
ON THE $p$-PRIMARY OBSTRUCTIONS TO FINDING A CROSS-SECTION
}

\author{
ROBERT RIGDON
}

ABSTRACT. Let $q: T \rightarrow B$ be a (weak) fibration, and let $p$ be an odd prime. In this paper, we show that the existence of a fiber-preserving map $A: T \rightarrow T$ having certain properties implies that the $p$-primary obstructions to a cross-section of $q$ vanish. Assuming that $q: T \rightarrow B$ has a cross-section, we prove a related theorem which bears on the problem of enumerating the homotopy classes of cross-sections of $q$.

1. Introduction. In this section we state the two main theorems (I and II) of this paper. These theorems are proved in $\$ 2$ and some applications are given in $\$ 4$.

Let $q: T \rightarrow B$ be a weak fibration (in the sense of Dold [5]) with fiber $F$ over $b_{0} \in B$. Assume that $F$ is $(n-1)$-connected, $n>1$, and that $B$ has the homotopy type of a connected CW complex and is $2 n$-coconnected. (Recall that a space $X$ is said to be $k$-coconnected if for every local system $\Gamma$ of abelian groups over $X$, we have $H^{q}(X ; \Gamma)=0, q \geq k$.) Let $O(T)$ be the single obstruction (see Larmore [7]) to a cross-section of $q: T \rightarrow B$.

Suppose that $A: T \rightarrow T$ is a fiber-preserving map covering the identity map of $B$, and let $a: F \rightarrow F$ be the restriction of $A$. Before stat ing Theorem I, we need the following

Definition. Let $p$ be an odd prime. If $G$ is an abelian group and $\alpha$ : $G \rightarrow G$ is a homomorphism, we say that $a$ has property $N I_{p}$ if there are positive integers $r$ and $s$, where $r$ is prime to $p$, such that $r\left(\alpha+1_{G}\right)^{s}$ : $G \rightarrow G$ is the zero map.

Theorem I. Assume that $B$ is $(l+1)$-coconnected where $l \leq 2 n-1$, and that $a_{*}: \pi_{i}(F) \rightarrow \pi_{i}(F)$ has property $N{ }_{p}$ for $i \leq l-1$ where $p$ is an odd prime. Then $O(T)$ has finite order prime to $p$ (or is zero).

Now assume that $q: T \rightarrow B$ has a cross-section and that $B$ is $(2 n-1)$ -

Received by the editors October 9, 1973 and, in revised form, December 19, 1973. AMS (MOS) subject classifications (1970). Primary 55G40, 55G45.

Key words and phrases. Fiber space, Stiefel bundle, cross-section, obstruction, Postnikov system. 
coconnected. Then $[B ; T]_{B}$, the set of homotopy classes of cross-sections of $q: T \rightarrow B$, has a natural affine group structure (see Becker [2]). This means that for any homotopy class $\lambda \in[B ; T]_{B},[B ; T]_{B}$ can be given a natural abelian group structure having $\lambda$ as its zero element. This group will be denoted by $\left([B ; T]_{B}, \lambda\right)$.

Composition with $A$ defines a function $A_{\#}:[B ; T]_{B} \rightarrow[B ; T]_{B}$ which is a homomorphism of affine group structures.

Theorem II. Assume that $B$ is $(l+1)$-coconnected where $l \leq 2 n-2$, and that $a_{*}: \pi_{i}(F) \rightarrow \pi_{i}(F)$ has property $N I_{p}$ for $i \leq l$ where $p$ is an odd prime. If $A_{\#}(\lambda)=\lambda$ and $A_{\#}\left(\lambda_{0}\right)=\lambda_{0}$ where $\lambda \neq \lambda_{0}$, then $\lambda$ has finite order prime to $p$ in $\left([B ; T]_{B}, \lambda_{0}\right)$.

Remarks. In Theorem I' below, we give an a lternate formulation of Theorem $I$ in terms of the obstruction theory associated with a factorization of $q: T \rightarrow B$ by $B$-principal fibrations.

As an application of Theorem I, we obtain a new proof of results of Copeland and Mahowald in [4] and Proposition 13.4 of Becker in [3]. (See Theorem 4.1 below.)

2. Proofs of the main theorems. We will make frequent use of the following simple lemma, the proof of which is left to the reader.

Lemma 2.1. Let $\{0\}=G_{m} \subset \dot{G}_{m-1} \subset \cdots \subset G_{0}=G$ be a finite filtration of the abelian group $G$ and assume that $\alpha$ is a filtration preserving endomorphism of $G$. Then a has property $N_{p}$ if and only if each of the maps $G_{i} / G_{i+1} \rightarrow G_{i} / G_{i+1}$ induced by $a, i=0, \cdots, m-1$, has property $N I_{p}$.

Proof of Theorem II. Let $\Gamma_{i}$ be the local system of abelian groups over $B$ defined by $\Gamma_{i}(b)=\pi_{i}\left(q^{-1}(b)\right)$ for $b \in B$. Set $L=\left([B ; T]_{B}, \lambda_{0}\right)$. Using the spectral seque nce from $[2, \S 4]$ or from $[7, \S 5]$, we obtain a filtration $\left\{\lambda_{0}\right\}=L^{l-n+1} \subset L^{l-n} \subset \cdots \subset L^{0}=L$, subgroups $C^{i} \subset D^{i} \subset H^{i}\left(B ; \Gamma_{i}\right)$, and is omorphisms $d^{i}: L^{i-n} / L^{i-n+1} \rightarrow D^{i} / C^{i}$ for $i=n, \cdots, l$. (This filtration could also be obtained using the methods of McClendon in [10].)

$A$ induces homomorphisms of local systems $\phi_{A}^{i}: \Gamma_{i} \rightarrow \Gamma_{i}$ which in turn induce homomorphisms $\left(\phi_{A}^{i}\right)_{*}: H^{i}\left(B ; \Gamma_{i}\right) \rightarrow H^{i}\left(B ; \Gamma_{i}\right)$. Since $A_{\#}\left(\lambda_{0}\right)=$ $\lambda_{0}$, the naturality of the spectral sequence implies that $A_{\#}\left(L^{i}\right) \subset$ $L^{i},\left(\phi_{A}^{i}\right)_{*}\left(C^{i}\right) \subset C^{i},\left(\phi_{A}^{i}\right)_{*}\left(D^{i}\right) \subset D^{i}$, and that the following diagram is commutative 


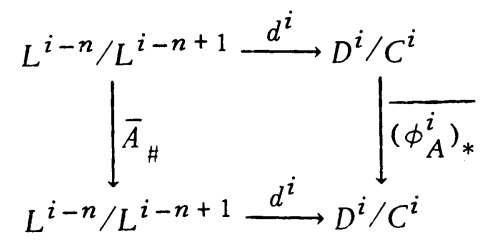

By hypothesis, $a_{*}=\phi_{A}^{i}\left(b_{0}\right): \Gamma_{i}\left(b_{0}\right) \rightarrow \Gamma_{i}\left(b_{0}\right)$ has property $N I_{p}$ for $i \leq l$. Applying Lemma 2.1 , we see that $\left(\phi_{A}^{i}\right)_{*}$ has property $N I_{p}$ for $i \leq l$, and that this implies that $A_{\#}: L \rightarrow L$ has property $N I_{p}$.

Let $r$ and $s$ be positive intergers with $r$ prime to $p$, such that $r\left(A_{\#}+1_{L}\right)^{s}$ is the zero map. Then $0=r\left(A_{\#}+1_{L}\right)^{s}(\lambda)=r 2^{s} \lambda$, so $\lambda$ has order prime to $p$.

Proof of Theorem I. Let $\bar{S}_{B}(T) \rightarrow B$ be the "fiberwise" unreduced suspension of $q: T \rightarrow B$. (The fibers of $\bar{S}_{B}(T) \rightarrow B$ are the unreduced suspensions of the fibers of $T \rightarrow B$, and $\bar{S}_{B}(T)$ is topologized as a quotient space of $T \times I$. By the appendix of [8], $\bar{S}_{B}(T) \rightarrow B$ is a weak fibration.) Let $s_{0}, s_{1}: B \rightarrow \bar{S}_{B}(T)$ be the south and north pole sections, respectively. $\left[B ; \bar{S}_{B}(T)\right]_{B}$ has a natural affine group structure; we assume from now on that $\left[B ; \bar{S}_{B}(T)\right]_{B}$ has been given the group structure having $\left[s_{0}\right]$ as its zero element.

The single obstruction $O(T)$ to a cross-section of $T \rightarrow B$ is the homotopy class of $s_{1}$ in $\left[B ; \bar{S}_{B}(T)\right]_{B}$. It was introduced for $s$ phere bundles by Becker in [3] (where it was called an Euler class relative to the sphere spectrum) and for general fibrations by Larmore in [7]. By [7, Theorem 4.3] $q: T \rightarrow B$ has a cross-section if and only if $O(T)$ is zero.

The map $A: T \rightarrow T$ induces $\bar{S}_{B}(A): \bar{S}_{B}(T) \rightarrow \bar{S}_{B}(T)$. Clearly $\bar{S}_{B}(A)_{\#}\left(\left[s_{i}\right]\right)=\left[s_{i}\right]$ for $i=0,1$. Hence Theorem I follows directly from The orem II.

The conventional method of attacking the existence problem for crossssections is via obstruction theory, and we now consider the implications of The orem I from the standpoint of obstruction theory. We first describe the factorization of a fibration by $B$-principal fibrations.

Recall that a space over $B$ is a space $Z$ together $w$ ith a continuous projection $Z \rightarrow B$, and that a $B$-sectioned space is a space over $B$ together with a canonical cross-section of the projection. (The projection and crosssection are normally suppressed from the notation.) If $Z_{1}$ and $Z_{2}$ are spaces over $B$, a map $f: Z_{1} \rightarrow Z_{2}$ is a map over $B$ if $f$ covers the identity map of $B$. If $f: Z_{1} \rightarrow Z_{2}$ is a map over $B$ and $Z_{2}$ is a $B$ - 
sectioned space, there is a notion of $B$-principal fibration induced by $f$ which generalizes the ordinary principal fibration. See $\mathrm{McClendon}$ ([10] or [9]) for the definition of $B$-principal fibration and a general exposition of the category of spaces over $B$.

By a factorization $\mathcal{F}$ of $q: T \rightarrow B$, we mean a commutative diagram

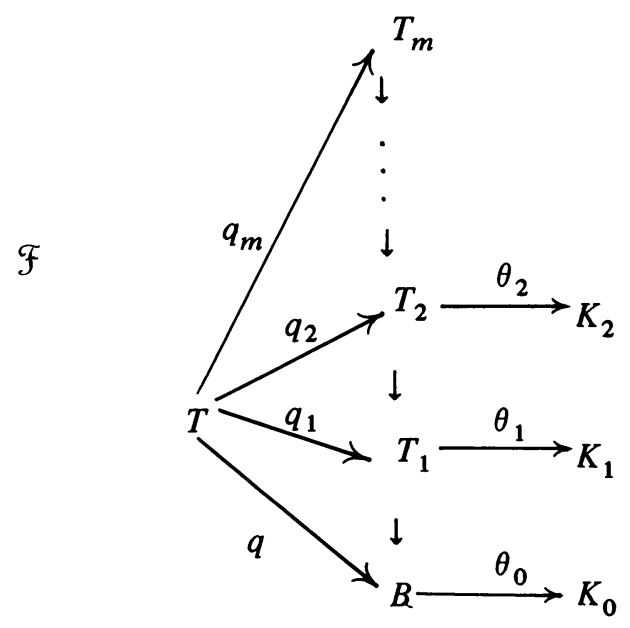

built up as follows. Each $K_{i}$ is a $B$-sectioned space such that the projection $K_{i} \rightarrow B$ is a weak fibration with $n$-connected fiber, each $\theta_{i}$ is a map over $B$, and $T_{i+1} \rightarrow T_{i}$ is the $B$-principal fibration induced by $\theta_{i}$.

A standard Moore-Postnikov factorization of $q: T \rightarrow B$ is one in which $K_{i}$ is an Eilenberg-Mac Lane space of type $\left(\Gamma_{n+i}, n+i+1\right)$ and $q_{i}$ is an $(n+i+1)$-equivalence.

The $i$ th obstruction of $\mathcal{F}$ is defined as follows

$$
O^{i}(\mathcal{F})=\left\{\left[\theta_{i} \circ g\right] \in\left[B ; K_{i}\right]_{B} \mid g: B \rightarrow T_{i} \text { is a cross-section of } T_{i} \rightarrow B\right\} .
$$

Observe that $\left[B ; K_{i}\right]_{B}$ has a group structure having the homotopy class of the canonical section as zero. Then $T_{i} \rightarrow B$ has a cross-section if and only if $O^{j}(\mathfrak{F})$ contains zero for $j<i$. Of course, the most useful factorizations are those which satisfy: $T_{m} \rightarrow B$ has a cross-section if and only if $q: T \rightarrow B$ has a cross-section. However, we do not restrict ourselves to such factorizations here.

We assume that the factorization $\mathcal{F}$ has the following property: There is a filtration $L^{m} \subset L^{m-1} \subset \ldots \subset L^{0}=\left[B ; \bar{S}_{B}(T)\right]_{B}$, subgroups Ind ${ }_{i} C$ $\left[B ; K_{i}\right]_{B}$, and monomorphisms $a_{i}: L^{i} / L^{i+1} \rightarrow\left[B ; K_{i}\right]_{B} /$ Ind $_{i}$ such that 
(1) $O^{j}(\mathcal{F})$ contains zero for $j<i$ if and only if $O(T) \in L^{i}$.

(2) If $O(T) \in L^{i}$, then $O^{i}(\mathcal{F})=\alpha_{i}\left(O(T)+L^{i+1}\right)$.

Theorem I'. If the hypotheses of Theorem I hold and $O^{j}(\mathcal{F})$ contains zero for $j<i$, then $O^{i}(\mathcal{F})$, as an element of $\left[B ; K_{i}\right]_{B} / \operatorname{Ind}_{i}$, has finite order prime to $p$ (or is zero).

Remark. For most factorizations $\mathcal{F}$, it is not difficult to verify the existence of a filtration of $\left[B ; \bar{S}_{B}(T)\right]_{B}$ having the above properties. Such filtrations exist for the standard Moore-Postnikov factorization by Larmore [7], and for the factorizations of [8] by their very construction. More generally, it follows from results of $\mathrm{McClend}$ on in [11] that the desired filtration exists for any factorization satisfying:

(1) Each $q_{i}$ is an $n$-equivalence.

(2) $\pi_{j}$ (fiber of $\left.K_{i} \rightarrow B\right)=0$ for $j \geq 2 n$, all $i$.

3. Property $\mathrm{NI}_{p}$. The following proposition is sometimes useful in verifying that a map satisfies the hypothesis of Theorem I or II. $\tilde{\pi}_{*}^{s}()$ denotes reduced stable hom otopy.

Proposition 3.1. Let $X$ be a connected pointed space and $f: X \rightarrow X$ a basepoint preserving map. Then $f_{*}: \tilde{\pi}_{i}^{s}(X) \rightarrow \tilde{\pi}_{i}^{s}(X)$ has property $N_{p}$ for $i \leq n$ if and only if $f_{*}: \tilde{H}_{i}(X ; \mathbf{Z}) \rightarrow \widetilde{H}_{i}^{i}(X ; \mathbf{Z})$ has property $N I_{p}$ for $i \leq n$.

Proof. Assume first that $f_{*}: \widetilde{H}_{i}(X ; \mathbf{Z}) \rightarrow \widetilde{H}_{i}(X ; \mathbf{Z})$ has property $N_{p}$ for $i \leq n$. By the naturality of the universal coefficient theorem and Lemma $2.1, f_{*}: \widetilde{H}_{i}(X ; G) \rightarrow \widetilde{H}_{i}(X ; G)$ has property $N I_{p}$ for each abelian group $G$ and each $i \leq n$. Let $\left\{E^{r}(X ; \mathcal{S})\right\}$ be the Atiyah-Hirzebruch spectral sequence converging to the quotients of a filtration of $\tilde{\pi}_{*}^{s}(X)$ with $E^{2}$ term $E_{*, *}^{2}(X ; \mathcal{S})=\widetilde{H}_{*}\left(X ; \pi_{*}(\mathcal{S})\right)$ where $\mathcal{S}$ is the sphere spectrum. (See [1, Chapter 7 l] for an exposition of the Atiyah-Hirzebruch s. s. )

Using natural ity of the spectral sequence $\left\{E^{r}(X ; \mathcal{S})\right\}$ together with Lemma 2.1, we see that $f_{*}: \tilde{\pi}_{i}^{s}(X) \rightarrow \widetilde{\pi}_{i}^{s}(X)$ has property $N I_{p}$ for $i \leq n$.

For the converse, let $\stackrel{G}{G}$ be the suspension spectrum of $X$, and let $K$ be a "nice" spectrum with $\pi_{0}(\mathcal{K}) \quad Z, \pi_{i}(\mathcal{K})=0$ for $i \neq 0$. Let $\left\{E^{r}(\mathcal{K} ; \tilde{\Xi})\right\}$ be the Atiyah-Hirzebruch spectral sequence converging to the quotients of a filtration of $\widetilde{G}_{*}(\mathcal{K})=\tilde{H}_{*}(X ; \mathrm{Z})$ with $E^{2}$ term

$$
E_{* *}^{2}(\mathcal{K} ; \xi)=\tilde{H}_{*}\left(\mathcal{K} ; \pi_{*}(\xi)\right)=\widetilde{H}_{*}\left(\mathcal{K} ; \tilde{\pi}_{*}^{s}(X)\right) \text {. }
$$


Now use naturality of this spectral sequence and apply Lemma 2.1 again.

If $X$ and $Y$ are spaces, let $X * Y$ be the usual join topologized as a quotient space of $X \times Y \times I$.

Corollary 3.2. Assume $Y$ is $(m-1)$-connected and $f: X \rightarrow X$ is a map. If $f_{*}: \tilde{\pi}_{i}^{s}(X) \rightarrow \tilde{\pi}_{i}^{s}(X)$ has property $N I_{p}$ for $i \leq n$, then $\left(f * 1_{y}\right)_{*}: \tilde{\pi}_{i}^{s}(X * Y)$ $\rightarrow \tilde{\pi}_{i}^{s}(X * Y)$ has property $N I_{p}$ for $i \leq n+m+1$.

Proof. This follows from Proposition 3.1, the Künneth formula and Lemma 2.1.

Remark. If $T \rightarrow B$ and $T^{\prime} \rightarrow B$ are weak fibrations, let $T{ }^{*}{ }_{B} T^{\prime} \rightarrow$ $B$ be their "fiberwise" join (see the appendix of [8]). Corollary 3.2 is useful for finding fiber preserving maps $T{ }_{B} T^{\prime} \rightarrow T{ }_{B}{ }_{B} T^{\prime}$ satisfying the hypotheses of Theorems I and II.

Proposition 3.3. Let $V_{m, k}$ be the Stiefel manifold of orthonormal $k$ frames in m-dimensional Euclidean space $\mathbf{R}^{m}$. Define $f: V_{m, k} \rightarrow V_{m, k}$ by $f\left(v_{1}, \cdots, v_{k}\right)=\left(-v_{1}, v_{2}, \cdots, v_{k}\right)$. Then for any odd prime $p, f_{*}: \pi_{i}\left(V_{m, k}\right)$ $\rightarrow \pi_{i}\left(V_{m, k}\right)$ has property $N I_{p}$ for

$$
\begin{array}{ll}
i \leq 2(m-k-1), & m \text { odd }, \\
i \leq \min (2(m-k-1), m-2), & m \text { even } .
\end{array}
$$

Proof. Half of this assertion is completely trivial since it is well known that if $m-k$ is odd, $\pi_{i}\left(V_{m, k}\right)$ is a 2-primary group for $k>1$ and $i$ in the above range. (See [6], p. 217.) The assertion is also obvious for $V_{m, 1}=S^{m-1}$.

To handle the remaining cases, let $\pi: V_{m, k} \rightarrow V_{m, k-1}$ be projection on the last $k-1$ vectors and let $j: S^{m-k} \rightarrow V_{m, k}$ be the inclusion of some fiber. We have a commutative diagram

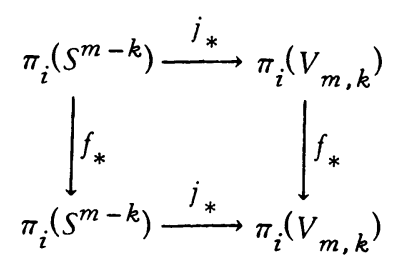

in which $j_{*}$ is a $\mathcal{C}_{2}$-epimorphism for $i$ in the range of 3.3 (where $\mathcal{C}_{2}$ is the Serre class of 2-primary groups). The assertions now follow from Lemma 2.1 . 
4. Applications. The following the orem is due essentially to Copeland and Mahowald [4]. (See also Becker [3].)

Theorem 4.1. Let $E \rightarrow B$ be an m-dimensional vector bundle and let $E_{k} \rightarrow B$ be the associated Stiefel bundle of orthonormal k-frames. If $B$ is $(l+2)-c o c o n n e c t e d$ where

$$
\begin{array}{ll}
l \leq 2(m-k-1), & m \text { odd }, \\
l \leq \min (2(m-k-1), m-2), & m \text { even },
\end{array}
$$

then the single obstruction $O\left(E_{k}\right)$ is zero modulo 2-torsion.

Proof. Define $A: E_{k} \rightarrow E_{k}$ by $A\left(v_{1}, \cdots, v_{k}\right)=\left(-v_{1}, v_{2}, \cdots, v_{k}\right)$. Then apply 3.3 and The orem $\mathrm{I}$.

Let $M$ be a compact smooth $m$-manifold. De note by $I\left(M, \mathbf{R}^{n}\right)$ (resp., $E\left(M, \mathbf{R}^{n}\right)$ ) the set of regular homotopy classes of smooth immersions (resp., isotopy classes of smooth embeddings) of $M$ in $\mathbf{R}^{n}$. $I\left(M, \mathbf{R}^{n}\right.$ ) (for $2 n>$ $3 m+1)$ and $E\left(M, \mathbf{R}^{n}\right)$ (for $2 n>3(m+1)$ ) have natural affine group structures. (See Becker [2].)

Let $C: \mathbf{R}^{n} \rightarrow \mathbf{R}^{n}$ be a linear transformation of determinant -1 . Composition with $C$ defines maps $C_{\#}: I\left(M, \mathbf{R}^{n}\right) \rightarrow I\left(M, \mathbf{R}^{n}\right)$ and $C_{\#}: E\left(M, \mathbf{R}^{n}\right)$ $\rightarrow E\left(M, \mathbf{R}^{n}\right)$ which are homomor phisms of affine group structures.

Theorem 4.2. Assume $g_{0}: M \rightarrow \mathbf{R}^{n}$ is an immersion such that $C_{\#}\left(\left[g_{0}\right]\right)=$ $\left[g_{0}\right]$ and $2 n>3 m+1$. Then $\left\{[g] \in I\left(M, \mathbf{R}^{n}\right) \mid C_{\#}([g])=[g]\right\}$, is a 2-primary subgroup of $\left(I\left(M, \mathbf{R}^{n}\right),\left[g_{0}\right]\right)$.

Theorem 4.3. Assume $g_{0}: M \rightarrow \mathbf{R}^{n}$ is an embedding such that $C_{\#}\left(\left[g_{0}\right]\right)=$ $\left[g_{0}\right]$ and $2 n>3(m+1)$. Then $\left\{[g] \in E\left(M, \mathbf{R}^{n}\right) \mid C_{\#}([g])=[g]\right\}$ is a 2-primary subgroup of $\left(E\left(M, R^{n}\right),\left[g_{0}\right]\right)$.

Proofs. Theorems of Haefliger and Hirsch (see [2, §9]) reduce the study of $I\left(M, \mathbf{R}^{n}\right)$ and $E\left(M, \mathbf{R}^{n}\right)$ to the study of the cross-sections of certain sphere bundles. Theorem II can then be applied. We give the details for 4.3 only.

Let $\Delta M$ be the diagonal in $M \times M$. The fixed point free involution mapping $(x, y) \in M \times M-\Delta M$ to $(y, x)$ defines a free $\mathrm{Z}_{2}$-action on $M \times M-$ $\Delta M$. Set $M \times M-\Delta M / Z_{2}=M^{*}$ and let $(M \times M-\Delta M)^{n-1} \rightarrow M^{*}$ be the $S^{n-1}$. bundle associated to the principal $\mathrm{Z}_{2}$-bundle $M \times M-\Delta M \rightarrow M^{*}$, where $Z_{2}$ acts on $S^{n-1}$ via the antipodal map. Haefliger's theorem says that for $2 n>3(m+1)$, there is a one-one correspondence $\tau: E\left(M, \mathbf{R}^{n}\right) \rightarrow$ $\left[M^{*} ;(M \times M-\Delta M)^{n-1}\right]_{M^{*}} ;$ moreover, $\tau$ is an isomrophism of affine group structures. 
Let $c: S^{n-1} \rightarrow S^{n-1}$ be the restriction of $C$ to $S^{n-1} \subset \mathbf{R}^{n}$. Then $c$ induces a bundle map $\bar{C}:(M \times M-\Delta M)^{n-1} \rightarrow(M \times M-\Delta M)^{n-1}$ and under the one-one correspondence $\tau, C_{\#}: E\left(M, \mathbf{R}^{n}\right) \rightarrow E\left(M, \mathbf{R}^{n}\right)$ becomes $\bar{C}_{\#}$. Hence 4.3 follows from Theorem II.

Remark. Let $\nu_{0}(g)$ denote the normal sphere bundle of the immersion $g: M \rightarrow \mathbf{R}^{n}$. If $C_{\#}([g])=[g]$, then it is easy to construct a bundle map $A$ : $\nu_{0}(g) \rightarrow \nu_{0}(g)$ covering the identity which has degree -1 on fibers. Hence Theorem I applies.

Remark. Theorems 4.2 and 4.3 can also be obtained by the methods of Becker in $[3, \S 11]$.

\section{REFERENCES}

1. J. F. Adams, Stable homotopy and generalized homology, Mathematics Lecture Notes, University of Chicago, Chicago, Ill., 1971.

2. J. C. Becker, Cohomology and the classification of liftings, Trans. Amer. Math. Soc. 133 (1968), 447-475. MR 38 \#5217.

3. - Extensions of cohomology theories, Illinois J. Math. 14 (1970), 551-584. MR 42 \#8476.

4. A. H. Copel and and M. Mahowald, The odd primary obstructions to finding a section in a $V_{n}$ bundle are zero, Proc. Amer. Math. Soc. 19 (1968), 1270-1272. MR 38 \#2780.

5. A. Dold, Partitions of unity in the theory of fibrations, Ann. of Math. (2) 78 (1963), 223-255. MR 27 \#5264.

6. D. Husemoller, Fibre bundles, McGraw-Hill, New York, 1966. MR 37 \#4821.

7. L. L. Larmore, Twisted cohomology theories and the single obstruction to lifting, Pacific J. Math. 41 (1972), 755-769.

8. M. Mahowald and R. Rigdon, Obstruction theory with coefficients in a spectrum, Trans. Amer. Math. Soc. (to appear).

9. J. F. McCle ndon, Higher order twisted cohomology operations, Invent. Math. 7 (1969), 183-214. MR 40 \#3541.

10. - Obstruction theory in fiber spaces, Math. Z. 120 (1971), 1-17. MR $45 \# 6002$.

11. - Reducing towers of principal fibrations, Nagoya Math. J. 54 (1974), 149-164.

DEPARTMENT OF MATHEMATICS, UNIVERSITY OF KENTUCKY, LEXINGTON, KENTUCKY 40506

Current address: Department of Mathematics, California State College, Dominguez Hills, California 90247 